\title{
Coulisses
}

Revue de théâtre

8 | Eté 1993

Varia

\section{La mise en scène de Cymbeline : une symphonie interculturelle}

Jean-Marc Fick

\section{OpenEdition}

1 Journals

Édition électronique

URL : http://journals.openedition.org/coulisses/2421

DOI : 10.4000/coulisses. 2421

ISSN : 2546-9460

Éditeur

Presses universitaires de Franche-Comté

Édition imprimée

Date de publication : 1 juillet 1993

Pagination : 11-14

ISSN : 1150-594X

\section{Référence électronique}

Jean-Marc Fick, "La mise en scène de Cymbeline : une symphonie interculturelle », Coulisses [En ligne], 8 | Eté 1993, mis en ligne le 15 mars 2019, consulté le 31 octobre 2019. URL : http:// journals.openedition.org/coulisses/2421; DOI : 10.4000/coulisses.2421

Ce document a été généré automatiquement le 31 octobre 2019.

Coulisses 


\title{
La mise en scène de Cymbeline : une symphonie interculturelle
}

\author{
Jean-Marc Fick
}

1 Il n'est pas question dans cette esquisse des préférences qui vont colorer la mise en scène globale de Cymbeline de rendre compte des divers signes visuels et sonores, tels que le costume, l'accessoire, l'éclairage, les sons et la musique et de régler les problèmes de leur résurgence à l'intérieur de l'espace-temps théâtral. On peut seulement affirmer, à la manière d'Artaud, que ces langages « investiront le spectateur » et qu'ils contribueront d'autant plus à l'intelligibilité de l'action qu'ils ont souvent une portée universelle. Quant à l'espace de jeu, il sera ce qui a été convenu : un plateau nu, où seront rendus visibles trois lieux, la terre, le ciel, l'enfer.

2 Pour coordonner les mises en scène partielles de Cymbeline, ou, en termes moins techniques, pour orchestrer la symphonie du spectacle à partir des chants particuliers, il convient d'agir à la fois sur la relation et sur le signe, deux plans de communication s'éclairant l'un l'autre. La poétique d'ensemble, qui tendra à exalter à la fois le singulier et le pluriel, se mettra au service de l'interculturel. De la symphonie doit rayonner un faisceau multisémiologique déchiffrable par tous et une harmonie propre à entraîner la communion festive.

\section{Un assemblage interactionnel}

Dans ce genre d'entreprise, il n'est pas envisageable de mettre à l'unisson les éléments disparates, sous peine de nuire à la spécificité de chaque production. Organiser, à l'inverse, un champ comparatif où les éléments sont mis en regard l'un de l'autre, comme par devoir de politesse excessif, produirait une satisfaction faible et stérile.

La problématique étant celle de la construction du récit, les prescriptions brechtiennes s'imposent à l'esprit. Evitons que le récit se déroule d'une manière linéaire, homogène, optons pour une poétique du récit décentré, se constituant par bonds, par montage discontinu. Si, dans les parties de la fable, apparaissent des « caractéristiques susceptibles 
de se contredire ", c'est pour multiplier et décomposer les points de vue sur l'événement et sur les personnages.

5 Le parti pris convient bien à Cymbeline, si l'on évite, toutefois, de le subordonner aux intentions qui sont celles de Brecht. Celui-ci vise en effet à étouffer l'émotion du spectateur pour le conduire à interposer son jugement au cours du déroulement narratif, aux points d'articulation plus précisément.

6 Or pour que la représentation reste un acte de communication, il est indispensable, tout en préservant ses capacités réflexives, de provoquer l'émotion du spectateur, sans pour autant le transformer en somnambule, grâce à des trucages illusionnistes permanents. Que le spectacle recèle à la fois des procédés propres à créer une illusion momentanée et des effets brechtiens n'est pas antinomique; cela correspond à une façon moderne de représenter la fable.

D'autre part, la distanciation, utilisée modérément, n'affadit ou ne brise pas forcément l'émotion. L'émotion vient surtout du rythme général, tour à tour soutenu et retenu, comme celui d'une vague qui ondule, se ramasse et repart de plus belle. L'émotion vient du spectacle d'une diversité préservée, mais organisée, harmonisée par le diapason. Il est essentiel que passe un souffle unique, même si ses aspirations et ses expirations suivent des rythmes différents. L'émotion émane de l'impression que donne un ensemble juste et organiquement clos. Orientons-nous alors vers un champ interactif qui mette les groupes en relation, tout en respectant leurs points de vue et leurs options. Les charnières entre les pièces fonctionneront à la fois comme des relais, des traits d'union, mais aussi comme des renforts de sens, enrichissant et l'avant et l'après. D'un rapport dynamique entre plusieurs identités qui se donnent mutuellement un sens, peut jaillir, semble-t-il, l'identité globale.

La Reine vue par le T.U. d'Edinburgh



Coulisses, 8 | Eté 1993 

dire de toucher le spectateur, de lui plaire et de le faire réfléchir. Mais pour amener davantage à la captatio benevolentiae, il faut mettre en œuvre d'autres moyens.

articulante et motrice, comme présence, matière, silhouette, mouvement.

\section{Une gestualité renforcée}

10 Une œuvre de Shakespeare, c'est, au premier contact, un texte et une langue. Bien que le comédien, par son corps et sa parole, les interprète, les barrages linguistiques subsistent. Mais comme le langage physique signifie de lui-même, on peut, non pas le rendre prioritaire, car il n'est pas question de subvenir l'autorité du verbe, mais lui donner plus de volume, afin de renforcer la dimension visuelle grâce à laquelle le spectacle devient plus lisible par l'ensemble des communautés.

11 Renforcer la gestualité consiste à favoriser tour à tour le hiératisme et le dynamisme. Les procédés sont connus des théâtres d'occident. La tragédie grecque, la tragédie classique et les recherches esthétisantes de "tableaux vivants", à références picturales, c'est une plastique qu'on cisèle. La turbulence orgiaque des satyres grecs, la farce médiévale, la commedia dell'arte, le burlesque, c'est une énergie qu'on aiguise.

12 Les figures corporelles collectives, les relais établis par les positions dans l'espace, le lien d'un geste à l'autre, les regards qui courent de l'un à l'autre, la gestuelle sobre, retenue au profit de la voix et du regard, se limitant à une fonction pragmatique ${ }^{1}$, tout cela combine des jeux de scène, donne une construction scénique à un texte et permet d'échapper à l'emprise du narratif linéaire.

13 Il en est de même des pantomimes chorégraphiques, de la succession de gestes rythmés, du déplacement aisé du corps dans l'espace. Le volume énergétique naît d'une expression gestuelle intempérée, débordante, de l'expressivité paroxystique des corps, sous le poids de la passion, par exemple.

14 Que le geste acquière une importance qui dépasse son statut de simple élément du langage théâtral n'est pas un but incongru, puisque le geste est, comme dit Régis Durand, « ce qui, par excellence, échappe au primat du discours verbal ».

\section{L'inattendu gestuel}

Dans la représentation théâtrale, tout est possible, excepté l'imitation de la banalité, le mimétisme uniformisateur. On courrait le risque de présenter une pâle doublure d'un réel sur lequel on ne se pose aucune question. Sur scène, le langage physique, ou kinésique, n'a pas à s'en tenir aux signes du quotidien pour être décodé. 


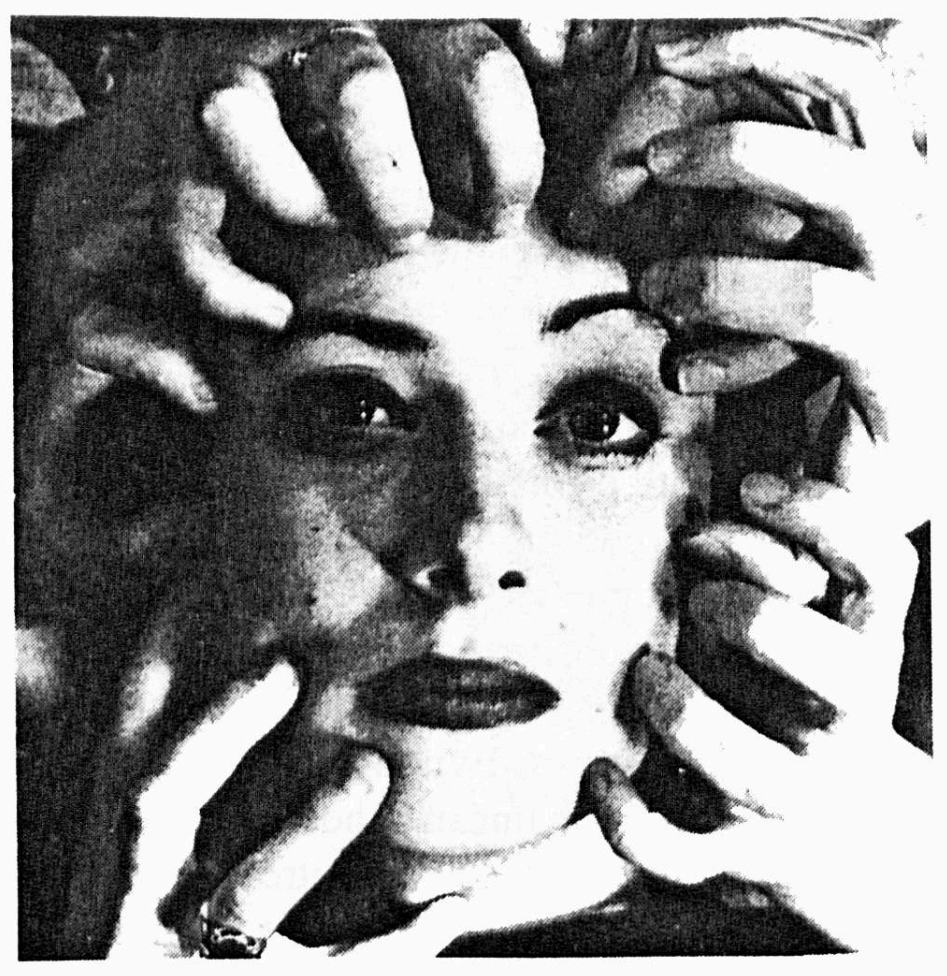

Sublimé, au contraire, il crée la surprise, suscite l'intérêt, éveille la curiosité ; il joue un rôle esthétique, symbolique et émotionnel, de première importance parce qu'il favorise le rapprochement communicatif. Peut-être parce qu'il retrouve sa magie d'origine, lorsque les gestes du petit être humain font naître les mots, que les mots éclosent de ses gestes.

La sublimation s'opère, si on le souhaite, grâce à des modes de présence corporelle choisis pour étonner le spectateur.

Si le geste est au service de la sensation, ou de l'objet, manipulé de manière utilitaire ou métaphorique, il fait aussi bonne alliance avec la parole.

Quand un personnage parle, mais qu'il a un geste comme suspendu ou étranger à la situation où il est, c'est un rien, une infime anomalie; or la phrase dite prend un léger déséquilibre ; elle est perçue dans son sens premier, mais quelque chose en détourne le sens profond. L'interrogation du spectateur se situe entre le geste et la phrase, mais il n'a pas vraiment le temps de se formuler cette interrogation, il reste en suspens lui aussi, comme le jeu, sa sensibilité en accord avec le climat, plutôt que sa démarche intellectuelle en accord avec le parcours du récit. Il y a là, peut-être, une façon d'abolir le jugement, et de provoquer une adhésion sensorielle, plus communicative peut-être que l'accord cérébral.

20 Une autre manière surprenante d'associer geste et parole est de rompre avec la traditionnelle simultanéité paraverbale. Le geste anticipe d'une fraction de seconde la parole, comme pour la faire naître, comme un coup de baguette magique fait s'envoler l'oiseau du chapeau. L'effet est accentué par le jeu corporel hyperbolique et redondant. Il y a là un exercice de style, fondé sur le principe de la distanciation brechtienne, qui a pour but d'étonner et de séduire le public. Il peut arriver que le message textuel, 
parasité par le geste, ne passe pas toujours intégralement, mais qu'importe, la parole en tire toujours profit.

21 A d'autres moments, le jeu de l'acteur tente de sortir du carcan formé par le texte. Par une exploration des dictions et de la kinesthésie qui vont à l'encontre de l'attendu, qui recherchent la rupture franche avec le conventionnel des intonations, des poses, des déplacements, il travaille "contre» le texte, pour fournir de l'inhabituel, voire de l'étrange, prélude éventuel à de nouvelles modalités de communication.

\section{La valeur du vide}

Théoriquement, pour parvenir à ces modalités de jeu et à cette disponibilité pour l'innovation, le comédien doit tenter de s'extraire de son identité, de son histoire, d'aller préalablement vers le "vide », afin que, loin des clichés envahissants mais aussi des états d'âme, des blocages et des petits problèmes quotidiens, son esprit soit bien disposé et que son corps devienne plus libre, conducteur d'une énergie transmissible au niveau du groupe.

Pour Peter Brook, les répétitions deviennent créatrices lorsque les habitudes, les idées reçues ont été expérimentées, rejetées, lorsque s'est fait le vide. Les membres du Living Théâtre affirment aussi qu' « on ne peut jouer qu'en partant de zéro, en se liquidant, en devenant une enveloppe vide" ou encore que "c'est après le point zéro que commence la partie essentielle du voyage $»^{2}$.

Inspirée des philosophies orientales, cette démarche tend à développer les échanges de chacun aux stimulations, aux intentions des autres (la transmission d'un son ou d'un geste par exemple d'un comédien à l'autre). Elle est le moyen de valoriser le corps. Une dynamique de l'énergie peut être ainsi créée, qui est la condition de communication entre les membres du groupe théâtral.

L'état de vacuité a déjà sans doute généré des productions originales chez les différents participants de Cymbeline. Il s'agira d'en retrouver le chemin lors de l'élaboration finale, au cours de laquelle il faudra rapidement mettre en place les jalons manquants.

La coloration et la clarté de la représentation dépend pour une bonne part de la gestualité recherchée. Elle n'apporte pas seulement un complément d'information sur les personnages, mais se révèle essentielle à leur identification et à la compréhension du message en général. Elle détermine aussi une lecture particulière de la pièce, qui s'inscrit dans les stratégies de séduction qu'exploite la mise en scène.

\section{Une poétique de la présence intense}

On l'a compris, Cymbeline développera sa puissance communicative, en activant les forces émotionnelles qui vibrent en chacun de nous. Cela sera aussi possible grâce à une matérialisation visuelle et plastique qui emplit l'espace de rythmes et de mouvements, et grâce à une efficacité spirituelle qui repose sur un langage charnel autant que sur la parole articulée. Nous pourrions prophétiser, comme Artaud: «Le chevauchement des images et des mouvements aboutira à des collisions d'objets, de silences, de cris, de rythmes ».

En fin de compte, pour rehausser le spectaculaire, qui touche tous les publics, il faut convoquer toutes les formes artistiques: texte, sonorités, cris, proférations, 
incantations, musiques, rythmes, silences, gestes, danse, chant, pantomime. Mais, au devant de tout, il y a le comédien, qui restera néanmoins le traducteur essentiel de Shakespeare. Que son jeu éveille les puissances mythiques du théâtre au point que la représentation retrouve le sens de rituels communs qui interpellent les peuples d'Europe.

Tous ces protocoles participent d'une conception de la dramaturgie qui veut que le centre de gravité de l'activité théâtrale ne se situe plus sur la scène ou dans l'œuvre seule, mais au point d'intersection de la scène et de la salle, ou mieux encore, à la jointure du théâtre et du monde. L'objectif est que Cymbeline réalise cet accord, et que la symphonie soit écoute, réponse, échange, plaisir, plaisir éphémère, comme les rêves, et rêve durable d'un monde soudé par le théâtre.

Dijon, mai 1993.

\section{Dessin réalisé par le T.U. d'Edinburgh}

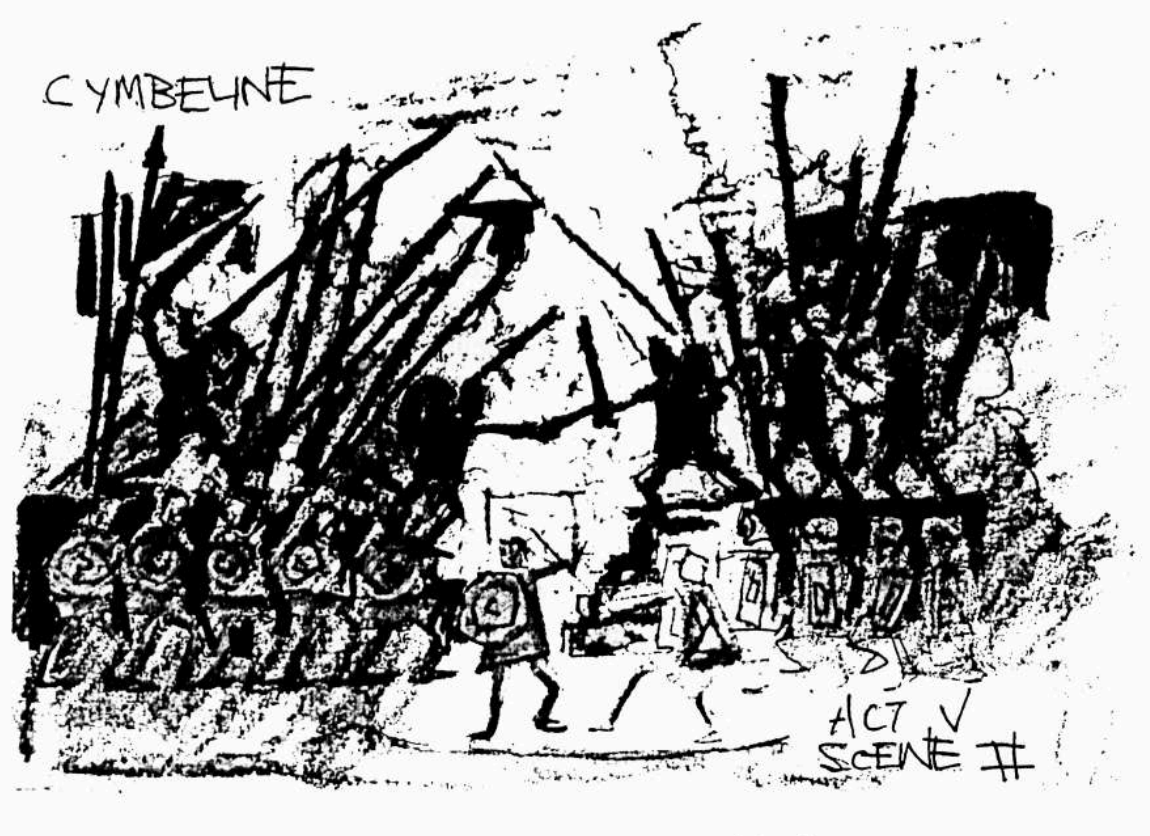

\section{NOTES}

1. que B. Koechlin ("Techniques corporelles et leur notation symbolique », Langages, $\mathrm{n}^{\circ} 10$, juin 1968, p. 36-47) appelle d' " efficacité technique ».

2. in J.J. Lebel, Entretiens avec Julian Beck et Judith Malina, Living Théâtre, Paris, Ed. Pierre Belfond, 1969. 